\title{
PREPARATION THE ROSE-LIKE HYDROPHOBIC SURFACE OF WOOD BASED MATERIALS BY SOFT LITHOGRAPHY
}

\author{
Zeyu Zhang, Bingru Shao, Fapeng Wang, Jiuyin Pang, Ling Su \\ BeIHUA University \\ China \\ (Received June 2020)
}

\begin{abstract}
To prepare hydrophobic wood with rose-like hydrophobic surface and avoid moisture damage to wood. In this paper, With polyvinyl alcohol (PVA) as the elastic mold, the microstructure of the rose petals was replicated on the wood surfaces by soft lithography. The soft lithography technique was used to modify the wood sur-face, transferring over it a rose-like topography, based on a micro/nano hierarchical structure using fresh rose as the template. The surface of poplar coated with polystyrene was reconstructed twice using $1,3,5$, 8 and 10\% PVA as templates, respectively. The results show that the average contact angle of poplar surface coated with polystyrene is more than $130^{\circ}$, that of fresh rose surface is about $140^{\circ}$, and that of untreated wood is about $60^{\circ}$. Therefore, the wood surface with polystyrene has obtained a similar structure to that of rose surface and has a certain hydrophobicity. In addition, the microstructures observed by means of SEM, showing rough surface structures with micro-nanopapillate hills on wood surfaces. Water droplets could easily roll down on such wood surfaces, exhibiting super-hydrophobic and low adhesion properties. The successful fabrication of rose-like wood provided a new direction for researches on the super-hydrophobic of wood, which could effectively prevent the damage of moisture to wood.
\end{abstract}

KEYWORDS: Hydrophobic wood, polyvinyl alcohol, polystyrene, soft lithography, rose petals.

\section{INTRODUCTION}

The surface of wood contains a large number of hydrophilic groups and pore structures, which will cause decay, cracking, and mildew in a long-term humid environment (Liu et al. 2015, Liu and Wang 2016, Lahtela and Karki 2015). The preparation of a hydrophobic wood can effectively avoid the damage of water to the wood (Ma 2018, Liu and Cao 2018), thereby increasing the service life of wood. The water droplets are not completely spread out on a smooth and flat ideal uniform solid surface but at a certain angle, as shown in Fig. 1a (Wang 2018). 
a
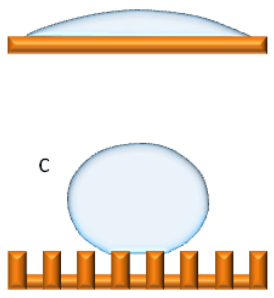

b

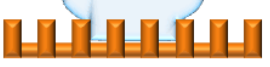

d

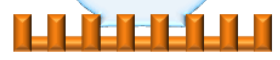

Fig. 1: The contact model of water drop on different rough surfaces.

But in fact, the surface has a certain roughness. The roughness affects the wettability of the material and determines the size of the contact angle. The wettability mainly depends on the surface energy and surface structure of the material (Wang et al. 2011, Jirous-Rajkovič et al. 2007, Kudela 2014). However, after hundreds of millions of years of evolution in nature, the special structure in the organism gives the living body a special function (Yang et al. 2016, Gao 2014), such as superhydrophobicity (Hou and Fang 2016, Wozniak et al. 2018), self-cleaning (Ma et al. 2013), hydrophilic (Wang et al. 2017a,b, Senarathna et al. 2020), low adhesion (Hou et al. 2019), etc. depending on the state of the water droplets on the superhydrophobic surface. The part where the droplet contacts the rough solid surface may be completely wetted, or it may contact the surface in a non-wetting mode with lower adhesion, or a transition state of the two, as shown in Figs. 1b-d. The rose surface is a surface with high adhesion and hydrophobicity (Ebert and Bhushan 2012). The petal surface has a dense micropapillary array with nano-folds. These regular micro-nano structures provide sufficient roughness for the hydrophobic effect (Zhang et al. 2018). According to the hydrophobic phenomenon existing in nature, it is bionic through chemical reagents to obtain a polymer film with multi-functional characteristics, which is covered on the surface of wood, so that the wood has a micro-nano surface with the same structure as rose petals, thus having the same as rose petal. The hydrophobic characteristics of the wood solve the defect of wood hydrophilicity, thus expanding the application range of wood.

Peng et al. (2013) adopted the template printing method, using fresh taro leaves as the mother board, so as to construct a structure with fine cavities on the surface, and then modified by dip coating method, and finally by poly-n-octadecylsiloxane The modification and modification of the nanosheets proved that the experimental results obtained significantly improved the hydrophobic properties. Lee et al. (2004) used hot pressing method to press polystyrene into porous alumina and waited for cooling to remove the alumina template, thus obtaining a hydrophobic polystyrene surface. Zhao et al. (2019) used chemical vapor deposition to obtain a hydrophobic film, but the cost of this method is relatively high and cannot be widely used in production.

In this paper, the template printing method is used to prepare the micro-nano structure of the rose-like surface on the wood surface using polyvinyl alcohol and polystyrene (Feng and Dou 2015, Pu et al. 2010). PS itself can reduce the surface energy of the substance to achieve a better hydrophobic effect on the wood surface (Lin et al. 2013, Cheng et al. 2014). The results show that using water contact angle, chemical analysis and other analysis methods to determine 
that the surface of the wood has the shape of a rose surface can effectively prevent moisture from entering the internal pores, thereby extending the service life of the wood and opening up new areas.

\section{MATERIAL AND METHODS}

Fresh rose petals, poplar specimens (purchased from Jilin City, Jilin Province, China), polyvinyl alcohol (PVA) reagents in different concentrations (the concentrations are 1, 3, 5, 8 and $10 \%$ ), self-made polystyrene (PS) were used for the experiment. Vacuum drying box: DZ-1BC was used for obtain the negative form of rose petals. Scanning electron microscope (SEM) was used for detect the surface morphology of poplar. The contact angle meter was used to measure the contact angle of the wood surface; the Energy Spectrometer: X-MaxN was used for elemental analysis ; the electric heating constant temperature water bath was used to heat the reagent.

\section{Preparation of polystyrene}

Take modification of polyoxyethylene alkylphenol ether by succinic acid (OS) and sodium lauryl sulfate (SLS) and dissolve them in a three-necked bottle, then drop styrene to obtain emulsifier (PE). At the same time, the temperature of the water bath was controlled at $75^{\circ} \mathrm{C}$, the $\mathrm{PE}$ that had already been prepared is added, and the potassium persulfate (APS) aqueous solution and Benzoyl peroxide (BPO) are added dropwise until the blue color disappeared, that is, PS emulsion is obtained.

\section{Mass fractions of PVA configuration}

The PVA powders of certain quality were separately taken into three bottles and placed in a water bath pot. The temperature was controlled between 88 and $95^{\circ} \mathrm{C}$. The solution with 1,3 , 5,8 and $10 \%$ mass fraction could be prepared by stirring until the PVA solution was clarified.

\section{Poplar samples were prepared by PVA/PS on the surface of rose petals}

First, trim the rose petals to a suitable size, and then stick them on the glass plate with milky glue, add the previously prepared PVA solution evenly on the surface of the rose flower, remove the air bubbles, and dry it in a vacuum drying oven at $60^{\circ} \mathrm{C}$ for about 2 hours (Fig.2) .
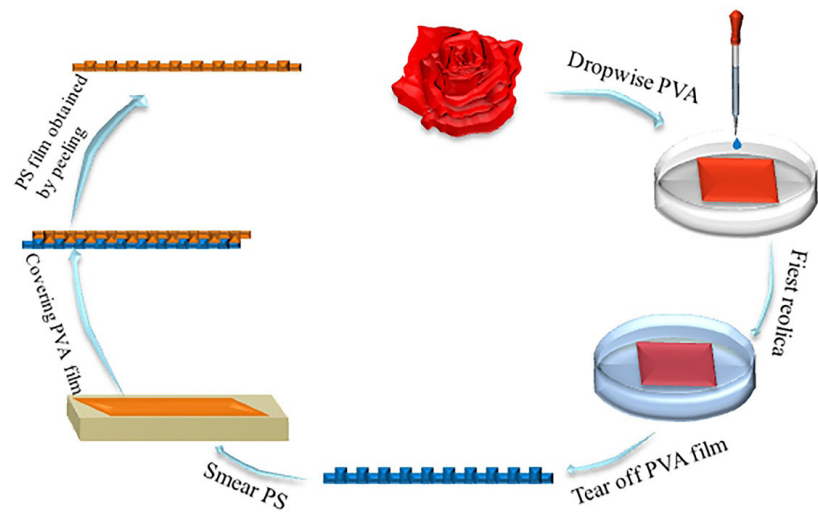

Fig. 2: The flow chart of the biomimetic preparation of rose structure surfaces. 
Take out and gently tear off the PVA soft film to get the negative type of rose petals. Then, the prepared PS was evenly coated on the poplar veneer with a glass rod, and then the PVA soft film was pasted on the poplar sample under a certain pressure, and put it at room temperature for 12 hours, peel off the PVA soft film, that is to say. A positive shape of rose petals was obtained on the surface of the poplar. Finally, the contact angle of water droplets on the poplar surface was measured by the contact angle tester, and the surface morphology of poplar was detected by the scanning electron microscope.

In the experiment, PVA has good film-forming properties and the mechanical properties of the film are excellent. PVA is covered on the rose petals to make it form a film opposite to the micro-nano structure of rose petals. PS has good corrosion resistance, and its solution is white liquid. Coating polystyrene on wood can solve the problem of dyeing white wood.

\section{Characterization of wood materials and treated wood surfaces}

The surface morphology of wood material and treated wood was observed by scanning electron microscopy (SEM). The microscopic image of a sample was obtained by enlarging the observation at different locations. The wettability of wood and treated wood was measured by OCA2 0 contact angle meter (German Dataphysics) at room temperature. The average value of contact angle was calculated and the final contact angle was obtained.

\section{RESULTS AND DISCUSSION}

\section{Morphology of water droplets on different sample surfaces}

Fig. $3 \mathrm{a}$ is a macro photo of water droplets on the surface of rose petals. It can be clearly seen that the water droplets are in a spherical state on the surface of the rose flower, which can explain that the surface of the rose petals has extremely strong hydrophobic properties.
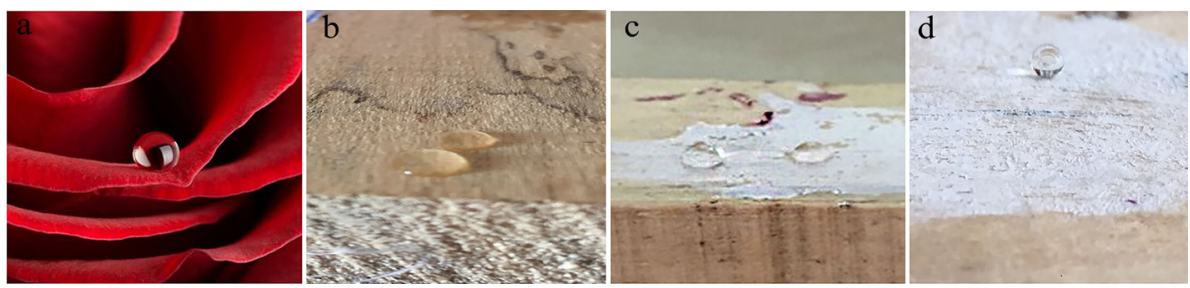

Fig. 3: Morphology of droplets on the surface of rose and wood specimen.

Fig. $3 \mathrm{~b}$ is a macro picture of water beads on the wood surface. The water beads are obviously wetted on the wood surface, and the contact angle is about $15.7^{\circ}$, which fully reflects that the wood is a hydrophilic material with a large number of hydroxyl groups and its hydrophobic performance is poor. Fig. $3 \mathrm{c}$ is a macro photo of water beads on wood coated with polyvinyl alcohol (PVA). The water beads are infiltrated on the surface, and the contact angle is about $73.24^{\circ}$, showing strong hydrophilic properties. Fig. $3 \mathrm{~d}$ is a picture of water drops on the surface of the bionic rose petals. The water drops are in a spherical state, and their contact angle is about $135.5^{\circ}$, which fully exhibits strong hydrophobic properties. It can be seen that after the wood is bionic, its contact angle has increased from $15.7^{\circ}$ to $135.5^{\circ}$, indicating that the surface of the wood after bionics has hydrophobic properties similar to rose petals. 


\section{Effect of different concentration of PVA solution on contact angle}

In Fig. 4a the contact angle of water droplets on the surface of wood material is only $15.7^{\circ}$, showing a hydrophilic state. In Fig. $4 \mathrm{~b}$ the contact angle of water droplets on wood surface treated by template method with $1 \%$ PVA film as master plate is $121.5^{\circ}$. The hydrophobicity of water droplets is improved obviously, which shows that this method has certain effect on improving the hydrophobicity of wood surface. Figs. 4c-e show the contact angle of wood surface treated with the concentration of 3\%,5\% and $8 \%$ of PVA film as water droplets. The contact angle shows a slow upward trend, but the difference is small, and the hydrophobic performance is relatively stable. Fig. $4 \mathrm{f}$ shows that the contact angle of the wood surface treated with $10 \%$ PVA as template is $135.5^{\circ}$. The water droplets are plump and round, showing super hydrophobicity and low adhesion, and the hydrophobicity is further improved. Fig. $4 \mathrm{~g}$ shows that the contact angle of rose surface is $143.5^{\circ}$.

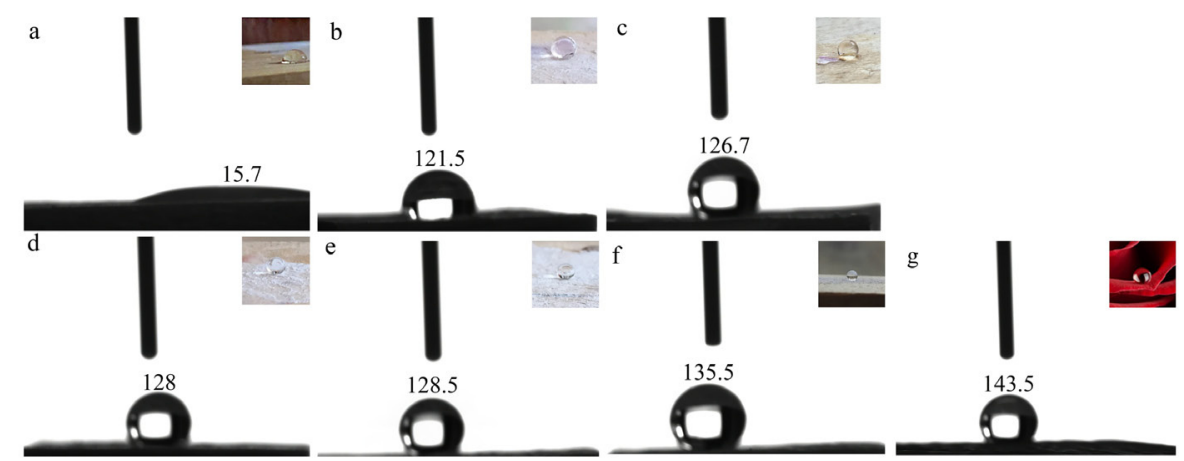

Fig. 4: Microscopic and macroscopic images of wood surface treated with water drops at different concentrations of PVA: (a) wood material surface, (b) 1\% PVA film, (c) 3\% PVA film, (d) 5\% PVA film, (e) $8 \%$ PVA film, (f) 10\% PVA film, $(g)$ rose surface.

It is also obvious from Fig. 5 that the effectiveness of PVA addition, and with the increase of PVA concentration, the contact angle of PS-coated wood surface increases gradually, and the 10\% PVA contact angle is closest to the rose surface, showing better hydrophobic effect.

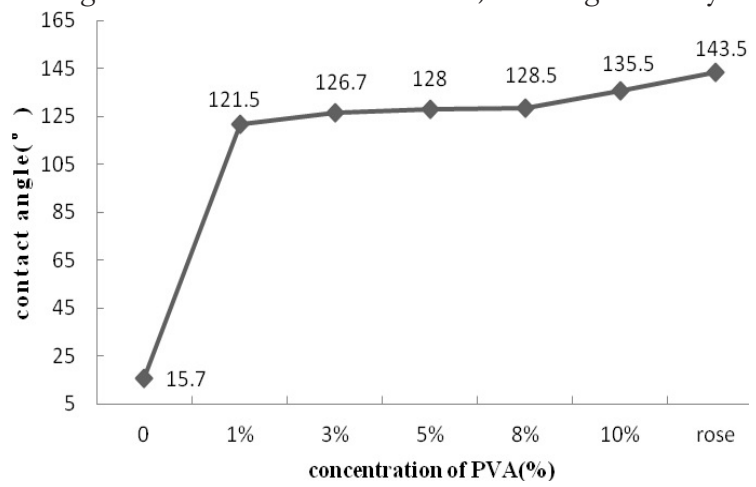

Fig. 5: Wood material, different concentrations of PVA film for the master treatment of wood and rose surface contact angle change trend. 
It is proved that the template printing method using PVA as template has made the same kind on the wood surface coated with PS. The surface structure of rose has similar hydrophobic effect with that of rose. Effect of wood surface hydrophobization caused using soft lithography was also reported by Ebert and Bhushan (2012), and surfaces exhibiting the so-called "petal effect" (super hydrophobicity with high droplet adhesion). Results obtained indicated that the micro and nano structures on the surface of roses were prepared by $\mathrm{ZnO}$ nanoparticles. The results showed that different concentrations of nanoparticles had different contact angles of high and low adhesion states, which proved that the hydrophobic performance of the experimental results was significantly improved.

In Figs. 6a,b can be seen that there are many porous holes on the surface of wood. The cell wall is composed of microcrystals, microfibrils and fibrils. These microcrystals and microcrystals, microfibrils and microfibrils, fibrils and fibrils are connected with each other and form a microcapillary system. Their inner surface is huge, so they have strong water absorption. Fig. $6 \mathrm{c}$ is a micrograph of PVA film obtained by template printing for the first time. The surface of PVA film has regular micro-nanostructure, which is the reverse of rose surface. Fig. $6 \mathrm{~d}$ is the result of secondary replica of the hydrophobic surface of rose-like wood. The surface is uneven, contrary to the PVA film, but it also has regular micro-nanostructure, which provides a certain roughness on the wood surface and improves the hydrophobic performance. However, compared with roses, the hydrophobicity of the surface similar to roses needs to be improved. This may be due to the partial relaxation of the applied stress in the PS film under a transient external load might also be responsible for some bit of smoothening of the replicated patterns.

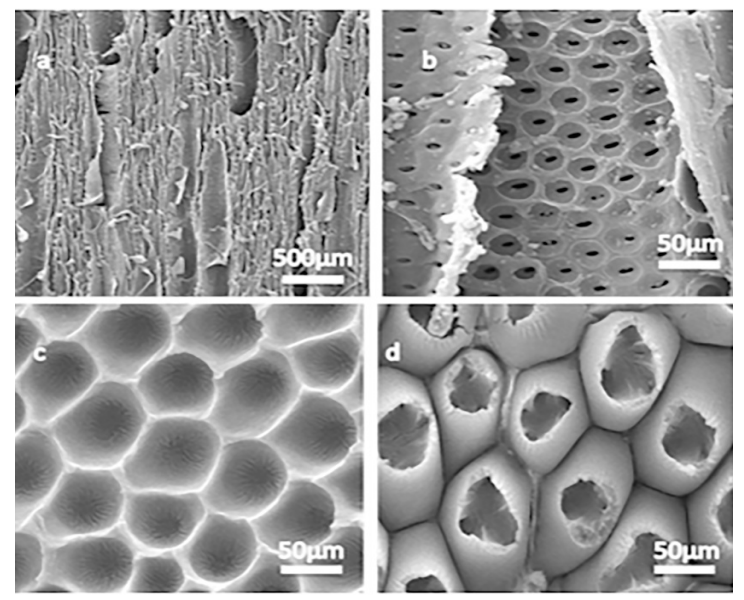

Fig. 6: SEM images of (a) and (b) wood surface, (c) PVA film, (d) a second replicated rose-like wood surface, (e) rose surface.

He et al. (2017) used soft printing technology to prepare the structural surface of similar Chinese rose on the surface of bamboo material. The bamboo material has relatively smooth surface microstructure, and the fiber texture is clearly visible. The bionic Chinese rose petal bamboo structure protruded outward, showing hemispherical shape, and arranged tightly with papillae of different sizes. The above evidence proves that biomimetic modification can improve the stability of bamboo, which also provides a reference for this paper. 

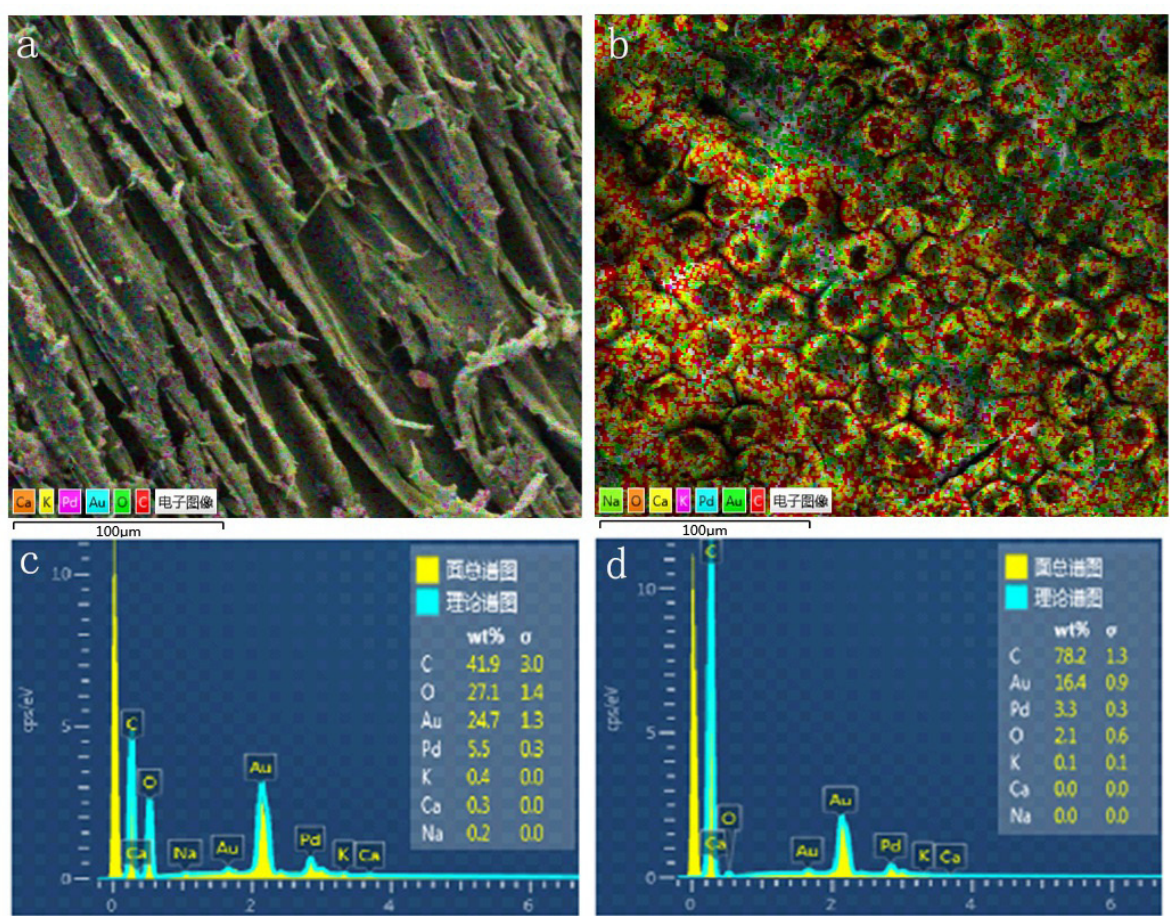

Fig. 7: The Energy dispersive spectrometer (EDS) spectrum of (a) wood surface, and (b) rose-like surface.

Figs. 7a,c are surface energy spectrum of wood. It can be seen that there are carbon, oxygen and a small amount of potassium, calcium and sodium on the surface of wood (gold and palladium come from coatings). The energy spectrum of the wood on the surface of rosette-like flowers in Figs. $7 \mathrm{~b}$,d show that there are only a large number of carbon and gold elements, a small amount of oxygen elements and no other elements, which indicates that the elements on the surface of rosette-like flowers come from wood and PS are not roses, confirming that the hydrophobic structure was effectively replicated on the rose-like wood surface.

\section{CONCLUSIONS}

Inspired by biology, this study successfully simulated the rough structure of rose surface on wood surface by template printing (soft printing technology) using rose as template and PVA of different concentration, which effectively prevented wood from damp damage. The study found that: (1) Two complex structures and their different surfaces can be observed by scanning electron microscopy (SEM). The first complex produces a structure opposite to that of a rose, while the second complex produces a rose-like structure on the surface of wood. (2) The water contact angle of the wood on the rose-like surface prepared by template printing is about $135.5^{\circ}$, very close to the contact angle of the rose surface. $1 \%$ PVA has very good hydrophobic effect. The contact angle of the wood surface is raised from 69 degrees to $121.5^{\circ}$, and with the increase of PVA concentration, the contact angle is also increasing. The experimental results show 
that 10\% PVA has the best hydrophobic effect. Contact angle closest to rose surface. (3) EDS can prove that the elements of wood on the surface of rose-like flowers come from wood rather than roses. (4) The successful preparation of rose-like hydrophobic wood surface proves that the hydrophobic properties of wood surface and other hydrophilic materials can be achieved by nanotechnology, which provides a new research direction for hydrophobic modification. (5) This study not only deepens the understanding of the hydrophobic mechanism of plant leaves in nature, but also provides a new direction for the study of water resistance and anti-corrosion of wood surface. Successful preparation of biomimetic hydrophobic wood can prolong the service life of wood, and broaden the application fields and prospects of wood industry.

\section{ACKNOWLEDGMENT}

We would like to acknowledge support from the National Natural Science Foundation of China (31800480), and Project of Jilin Provincial Development and Reform Commission (2021C041-6).

\section{REFERENCES}

1. Cheng, X.L., Wang, S.T., Hong, B.S., Zhou, Q. 2014: Study on improving hygroscopicity of ammonium nitrate coated with polystyrene. Adhesion 35(04): 76-78+87.

2. Ebert, D., Bhushan, B., 2012: Wear-resistant rose petal-effect surfaces with superhydrophobicity and high droplet adhesion using hydrophobic and hydrophilic nanoparticles. Journal of Colloid and Interface Science 384(1): 182-188.

3. Feng, Y.Y., Dou, X.Q. 2015: Study on micro-nanostructure and antimicrobial adhesion of biomimetic rose petals. Biotechnology World 2: 181.

4. Gao, N., 2014: Construction and anti-fouling performance of stable bionic hydrophilic surface . Northwest University. Shan Xi, 90 pp.

5. He, X.W., Fu, S.Y., Dai, Y.P., Jin, C.D., Wang, F.P., 2017: Research on surface characteristics of bamboo with bionic super-hydrophobic Chinese rose/ $\mathrm{TiO}^{2}$. World Bamboo and Rattan 15(05): 11-15.

6. Hou, L.X., Fang, L., 2016: Progress in preparation and application of superhydrophobic surfaces. Chemical Bulletin 79(10): 897-904.

7. Hou, D., Li, T., Chen, X., He, S., Ren, Z.J., 2019: Hydrophobic nanostructured wood membrane for thermally efficient distillation. Science Advances 5(8): eaaw3203.

8. Jirouš-Rajkovič, V., Bogner, A., Mihulja, G., Vrsaljko, D., 2007: Coating adhesion and wettability of aged and preweathered fir wood and pine wood surfaces. Wood Research 52(2): 39-48.

9. Kudela, J., 2014: Wetting of wood surface by a liquids of a different polarity. Wood Research 59(1): 11-24.

10. Liu, M., Wu, Y.Q., Qing, Y., Tian, C.H., Jia, S.L., Rosa, Li, X.G., 2015: Advances in biomimetic superhydrophobic functional modification of wood. Functional Materials 46(14): 14012-14018.

11. Liu, F., Wang, C.Y., 2016: Biomimetic superhydrophobic functionalization of wood. Science and Technology Report 34(19): 389-396.

12. Lee, W., Jin, M.K., Yoo, W.C., Lee, J.K., 2004: Nano-structuring of a polymeric substrate with well-defined nanometer-scale topography and tailored surface wettability. Langmuir 20(18): 7665-7669. 
13. Lin, G.Q. Li, P., Xiong, X.F., Lu, G.Q. Wang, X.H., Qiu, L.Z., 2013: Fabrication of high performance flexible thin film transistors by different surface modifications. Journal of Luminescence 34(10): 1392-1399.

14. Liu, Z., Cao, J.Z., 2018: Fabrication of superhydrophobic wood surface with a silica/silicone oil complex emulsion. Wood Research 63(2): 353-364.

15. Lahtela, V., Karki, T., 2015: Determination and comparison of some selected properties of modified wood. Wood Research 60(5): 763-772.

16. Ma, Y.Z., 2018: Preparation and multifunction of super-hydrophobic surface. Contemporary Chemical Research 8: 148-150.

17. Ma, R., Wei, Y., Guo, H., Yang, F., Qian, F., 2013: Preparation and characterization of a new bionic-recreation superhydrophobic surface. Chinese Journal 58(14): 1326.

18. Peng, P.P., Ke, Q., Zhou, G., Tang, T., 2013: Fabrication of microcavity-array superhydrophobic surfaces using an improved template method. Journal of Colloid and Interface Science 395: 326-328.

19. Pu, X., Ge, J.F., Chen, C.C., 2010: Research progress on biomimetic superhydrophobic surface with micro-nano hierarchical structure. Guangdong Chemical Industry 37(5): $25-28+40$.

20. Senarathna, R.M.D.M., Wanniarachchi, W.K.I.L., Jayawardhana, S., 2020: Replication of the surface wettability of plant leaves with different surface morphologies using soft lithography. International Journal of Nanoscience 19(3): 1950018.

21. Wang, N., Zhao, Y., Jiang, L., 2011: Bioinspired multi-scale micro/nanostructured materials. Journal of Chemistry of Colleges and Universities 32(3): 421-428.

22. Wang, F.P., 2018: Biomimetic preparation of plant leaf micro-nano structure and its properties by bamboo surface deposition method. Zhejiang A\&F University: Pp 8-11.

23. Wang, F.P., Li, S., Wang, L., 2017a: Fabrication of artificial super-hydrophobic lotus-leaflike bamboo surfaces through soft lithography. Colloids and Surfaces A: Physicochemical and Engineering Aspects 513: 389-395.

24. Wang, F.P., Wang, L., Wu, H.P., Pang, J., Gu, D., Li, S., 2017b: A lotus-leaf-like SiO2 superhydrophobic bamboo surface based on soft lithography. Colloids and Surfaces A: Physicochemical and Engineering Aspects 520: 834-840.

25. Wozniak, M., Ratajczak, I., Lis, B., Krystofiak, T., 2018: Hydrophobic properties of wood treated with propolis-silane formulations. Wood Research 63(3): 517-524.

26. Yang, W.L., Ji, X.B., Xu, J.L., 2016: Super hydrophilic surface from nature to bionics to practical application. Chemical Progress 28(6): 763-772.

27. Zhang, Y.W., Jingran, B., Wang, S.Q., Cao, Q.Q, Li, Y., Zhou, J.H., Zhu, B.W., 2018: Functional food packaging for reducing residual liquid food: thermo-resistant edible superhydrophobic coating from coffee and beeswax. Journal of Colloid and Interface Science S0021979718310762.

28. Zhao, Y., Song, B., Cui, X., Ren, Y., Yue, W., Wang, Y., 2019: High electrocatalytic reduction using $\mathrm{ZnS}$ micropolyhedron: Direct sulfuration of ZIF-8 film on conducting glass by chemical vapour deposition. Materials Letters 250: 193-196. 
Zeyu Zhang, Bingru Shao, Fapeng Wang, Jiuyin Pang*, Ling su* Beihua University Yifu building, East Campus of North China University I32OI3 JiLIN

China

*Corresponding authors: pangjiuyin@163.com and sulingling2007@163.com 